\title{
Gesundheitsschäden bei Fluglärm
}

\section{Hans Göschke}

PD Dr. med., Vorstandsmitglied Ärztinnen und Ärzte für Umweltschutz AefU

\author{
Die Eidgenössische Lärmkommission hat einstimmig entschieden, dass unsere \\ Fluglärm-Grenzwerte zu hoch angesetzt sind, weil sie nicht vor Gesundheits- \\ schäden schützen. Dazu wurden im Nationalrat zwei Vorstösse eingereicht. Für \\ die Frühjahrssession vom kommenden März ist eine parlamentarische Initiative \\ zur Verlängerung der Nachtflugruhe traktandiert.
}

Weit über 100 Studien befassten sich bisher mit dem Zusammenhang zwischen Fluglärm und einer Vielzahl von Krankheiten. Schlafstörungen, Entwicklungsstörungen bei Kindern und Herzkreislauferkrankungen sind die wichtigsten und bestuntersuchten Gesundheitsschäden, welche bei Fluglärm vermehrt auftreten. Alle wurden bei Fluglärmpegeln beobachtet, wie sie im Umkreis unserer Landesflughäfen gemessen werden. Bei gleicher Lautstärke ist das Störpotential von Fluglärm mehrfach höher als jenes von Bahn- oder Strassenlärm [1, 2].

\section{Schlafstörungen}

Der menschliche Organismus reagiert im Schlaf auf Lärm viel empfindlicher als im Wachzustand. Die hohe Empfindlichkeit des hormonalen und neuralen Systems in der Nacht ist ein biologisches Erbstück. Sie war für unsere Urahnen ein überlebenswichtiges Alarmsystem.

Die Stresshormone Kortisol, Adrenalin und Noradrenalin steigen im Schlaf schon ab Mittelpegeln von 35 Dezibel (dB) an [2-4]. Erlaubt sind jedoch im Jahresschnitt Fluglärm-Mittelpegel bis $55 \mathrm{~dB}$ von 2223 Uhr und $50 \mathrm{~dB}$ von 23-24 Uhr sowie von 5-6 Uhr. Damit wird bei offenen Fenstern der erwähnte Schwellenwert von $35 \mathrm{~dB}$ deutlich überschritten.

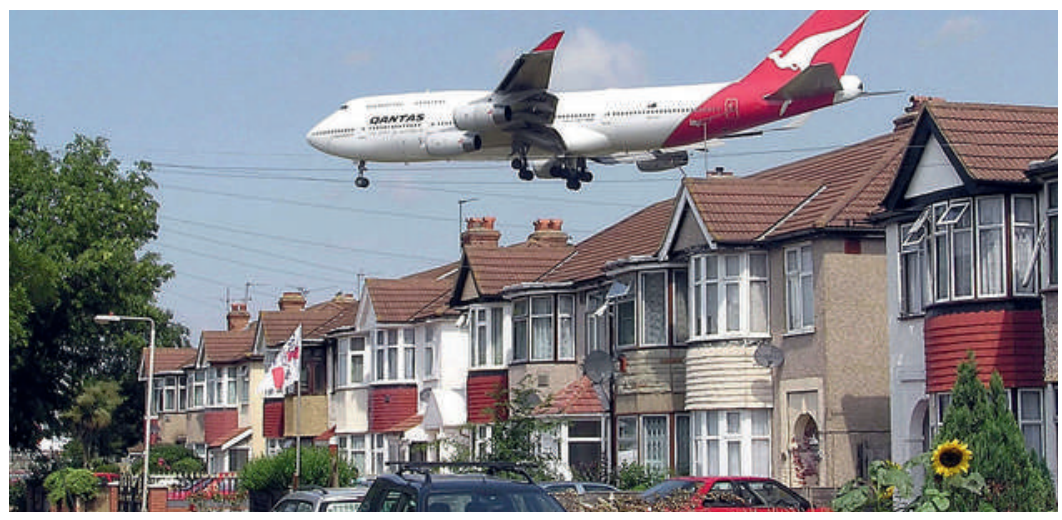

Die Stresshormone führen zu Blutdruckanstieg und Verschlechterung der Schlafstruktur - auch ohne Aufwachen [2-6]. Auch wer sich subjektiv nicht gestört

In Fluglärmzonen wurden signifikante Lerndefizite bei Schulkindern nachgewiesen.

fühlt, erleidet eine Verschlechterung der Schlafqualität [2]. Tiefschlaf und Traumschlaf werden schon $a b$ Mittelpegeln von 40 dB verkürzt [2, 4]. Der Tiefschlaf ist Voraussetzung für die körperliche, der Traumschlaf für die seelische Erholung. Die ungenügende nächtliche Erholung führt zu reduzierter Reaktions-, Konzentrations-, und Leistungsfähigkeit am Tag.

Der gesetzliche Grenzwert von $55 \mathrm{~dB}$ für die Stunde von 22 bis 23 Uhr ist ein Jahresmittelpegel. Dieser erlaubt zum Beispiel eine tägliche Lärmspitze von $81 \mathrm{~dB}$ oder zwei Spitzen von $78 \mathrm{~dB}$, die bei offenen Fenstern Jede und Jeden aus dem Schlaf reissen. Aufwachreaktionen erfolgen schon bei Lärmspitzen ab $50 \mathrm{~dB}[4,6]$. Eine Gewöhnung an Fluglärm gibt es nicht: Die Veränderungen von Stresshormonen, Blutdruck und Schlafstruktur bleiben auch nach jahrelanger Exposition bestehen $[2,5]$.

\section{Am meisten schadet Fluglärm den Kindern}

Laut WHO stellen Kinder eine besondere Risikogruppe für nächtlichen Fluglärm dar. Die bei Erwachsenen beschriebenen Schlafstörungen gelten noch vermehrt für Kinder. Dazu kommen aber noch zwei für Kinder typische Schädigungen.

\section{Lernstörung durch Schlafstörung}

Im Schlaf erfolgt die Überführung von Inhalten des Frischgedächtnisses ins Langzeitgedächtnis, ein Vorgang, der für den Lernprozess unentbehrlich ist. In Fluglärmzonen wurden signifikante Lerndefizite bei Schulkindern nachgewiesen [2, 7-10], genannt Lernstö- 
rung durch Schlafstörung. Als Mechanismus wird ein Lärm-induzierter Kortisolanstieg angenommen. Es ist gelungen, durch Kortisolverabreichung - in Abwesenheit von Lärm - die Lernstörung zu simulieren. Durch die Betriebszeiten etwa des Basler Flughafens von 5 bis 24 Uhr wird die 10- bis 11-stündige Nachtruhe der Primarschüler zwischen 20 und 7 Uhr öfter halbiert.

\section{Learned Helplessness}

Dem Fluglärm gegenüber ausgeliefert und machtlos zu sein, ist zunächst eine subjektive Erfahrung; aber objektiv bedeutet dies Resignation, Demotivation und mit dem gebräuchlichen Fachausdruck learned helplessness (erlernte, anerzogene Hilflosigkeit).

Schulkinder am alten und neuen Standort des Flughafens München sowie in einer Kontrollregion wurden einerseits ein halbes Jahr vor, andrerseits ein halbes und anderthalb Jahre nach der Flugplatzverlegung getestet [8]. Mit folgendem Ergebnis: Resignation und Demotivation beschränkten sich nicht auf den Fluglärm, sondern übertrugen sich auch auf andere Lebensbereiche, sie wurden zur Lebenseinstellung. Beide dauerten nach der Verlegung des Flughafens noch mindestens eineinhalb Jahre an.

Weitere Untersuchungen zeigten eine schlechtere psychosoziale Gesundheit und eine hoch signifikante Hyperaktivität der betroffenen Kinder. Fluglärm war wiederum deutlich schädlicher als gleich lauter Strassenlärm.

In keiner Studie konnte eine "Gewöhnung» an den Fluglärm nachgewiesen werden, die Schädigungen blieben in Langzeitstudien unverändert.

\section{Herzkreislauferkrankungen}

Fluglärm erhöht das Risiko von Herzkreislauferkrankungen. Dazu gehören Bluthochdruck, Herzinfarkt, Herzversagen und Hirnschlag. Was internationale Studien gezeigt haben, ist auch für die Schweiz belegt. Eine gemeinsame Untersuchung der Universitäten Basel und Bern hat aufgezeigt, dass tödliche Herzinfarkte um Schweizer Flughäfen bis zu $48 \%$ häufiger sind als in Fluglärm armen Gebieten [11]. Am grössten war die Gefahr für Personen, die 15 und mehr Jahre in Flughafennähe gewohnt hatten und einem durchschnittlichen Lärm von 60 und mehr Dezibel dn* pro 24 Stunden ausgesetzt waren. Es besteht folglich eine eindeutige Dosis-Wirkungsbeziehung zwischen Fluglärm und Herzinfarkt: Je grösser der Fluglärm und je länger die Exposition, desto häufiger die tödlichen Herzinfarkte. Die Autoren betonen, dass es keinen eindeutigen Schwellenwert gibt, ab dem das Infarktrisiko zunimmt. Vielmehr steigt das Risiko schon bei wesentlich tieferen Lärmpegeln als $60 \mathrm{~dB} d \mathrm{n}^{*}$ an. Nach Greiser ist dieses Risiko schon bei mittleren Fluglärmpegeln von $50 \mathrm{~dB}$ signifikant erhöht [13], also weit unterhalb der Schweizer Lärmgrenzwerte.

Mehr als ein Dutzend internationale Studien haben den Zusammenhang zwischen Fluglärm und Herzkreislauferkrankungen aufgezeigt [6, 11-15]. Dies obwohl sie von verschiedenen Autoren, in verschiedenen Ländern und mit unterschiedlichem Studiendesign erfolgten. Die Resultate erreichten dadurch einen hohen Grad von Konsistenz. Dies spricht dafür, dass der Zusammenhang kausal ist [13, 15]. «Ein ursächlicher Zusammenhang (Kausalzusammenhang) zwischen Fluglärm, vor allem in der Nacht, und dem Auftreten von Herzkreislaufkrankheiten wird in der epidemiologischen Wissenschaft anerkannt» [13].

\section{Literatur}

1 Miedema HME, Voss H. Exposure-response relationships for transportation noise. J. Accoust. Soc. Amer. 1998;104:3432.

2 Good practice guide on noise exposure and potential health effects. European Environment Agency, European Union. Technical Report No 11; 2010.

3 Maschke C, Arndt D. Ising H. Nächtlicher Fluglärm und Gesundheit. Ergebnisse der Labor- und Feldstudien. Bundesgesundheitsbl. 1995;38:130.

4 Maschke C, Ising H, Hecht K. Schlaf - nächtlicher Verkehrslärm Stress - Gesundheit. Teil 1: Grundlagen; Bundesgesundheitsbl. 1997; 40. 1. Teil 2: Aktuelle Forschungsergebnisse Bundesgesundheitsbl. 1997; 40:3

5 Ising H, Kruppa B. Zum gegenwärtigen Erkenntnisstand der Lärmwirkungsforschung. Umweltmed Forsch Prax. 2001;6;181.

6 Haralabidis AS, Dimakopoulou K, Jarup L et al. Acute effects of night-time noise exposure on blood pressure in populations living near airports. Europ Heart J. 2008;29:658.

7 Banatvala J. Unhealthy airports. Lancet. 2004;364:646.

8 Bullinger M, Hygge S, Evans GW, Meis M, von Mackensen S. The psychological cost of aircraft noise for children. Zbl Hyg Umweltmed. 1998/99;202:127.

9 Stansfeld S, Halpes M, Brown B. Aircraft and road traffic noise and children's cognition and health: a cross-national study. Lancet. 2005;365:1942.

10 Clark C, Martin R,van Kempen E, Stansfeld SA. et al. Exposureeffect relations between aircraft and road traffic noise exposure at school and reading comprehension: the RANCH project. Am J Epidemiol. 2006;163:27.

11 Huss A, Spöri A, Egger M, Röösli M. Aircraft noise, air pollution, and mortality from myocardial infarction. Epidemiology. 2010;21:829.

12 Correia A W, Peters JL,Levy JI, Melly S, Dominici F. Residential exposure to aircraft noise and hospital admission for cardiovascular disease: multi-airport retrospective study. Brit Med J. 2013;347:11.

13 Greiser E, Greiser C. Risikofaktor nächtlicher Fluglärm. Abschlussbericht über eine Fall-Kontroll-Studie zu kardiovaskulären und psychischen Erkrankungen im Umfeld des Flughafens Köln-Bonn. Umweltbundesamt, Schriftenreihe Umwelt und Gesundheit 01/2010.

14 Jarup L, Babisch W, et al: Hypertension and exposure to noise near airports: The HYENA Study. Environ. Health Prospect. 2008;116:329.

15 Kaltenbach M, Maschke C. Er macht doch krank. Die Datenlage verdichtet sich, dass Lärm zu vermehrtem Auftreten von Hypertonie, Herzinfarkt und Schlaganfall führt. Dt Ärzteblatt. 2011;108:2266 